\title{
Hybrid Quantum Mechanical - Quasi-classical Model for Evaluating Ionization and Stripping Cross Sections in Atom-Ion Collisions *
}

\author{
Igor D. Kaganovich ${ }^{\dagger}$, Edward A. Startsev, Ronald C. Davidson \\ Plasma Physics Laboratory, Princeton University, Princeton, New Jersey, 08543 USA
}

\begin{abstract}
Ion-atom ionization cross sections are needed in many applications employing the propagation of fast ions through matter. When experimental data or full-scale theoretical calculations are non-existent, approximate methods must be used. The most robust and easy-to-use approximations include the Born approximation of quantum mechanics and the quasi-classical approach utilizing classical mechanics together with the Bohr-Sommerfeld quantization rule. A hybrid method has been developed for calculation of the ionization and stripping cross sections of ions or atoms by fast ions by combining the Born approximation of quantum mechanics and the quasi-classical approach in the regions of impact parameters in which they are valid, and summing the results to obtain the total cross section. The results of the modified approach agree better with the experimental data than either the Born approximation or the quasi-classical approach, applied separately. A new scaling formula for the ionization and stripping cross section of atoms and ions by fully stripped projectiles has been also proposed. The scaling has no fitting parameters and has been checked against 11 different pairs of projectile and target atoms. All of the experimental data overlap on a single curve in the proposed scaled coordinates.
\end{abstract}

\section{INTRODUCTION}

Ion beams lose electrons when passing through a background gas in accelerators, beam transport lines, and target chambers. As a result, the ion confinement time and beam focusability are decreased. An unwanted electron population, produced in ion-atom collisions, may also lead to the development of collective two-stream instabilities. Therefore, it is important to assess the values of ion-atom ionization cross sections $[1,2]$. In contrast to ionization cross sections by electrons and protons, where experimental data or theoretical calculations exist for practically any ion and atom, the knowledge of ionization cross sections by fast complex ions and atoms is far from complete [3]. We have recently investigated theoretically and experimentally the stripping of more than 18 different pairs of projectile and target particles in the range of 3-38 MeV/amu to study the range of validity of both the Born approximation and the classical trajectory calculation $[4,5]$. In most cases, both approximations give similar results. However, for fast projectile velocities and low ionization potentials, the classi-

\footnotetext{
* Research supported by the U. S. Department of Energy.

† ikaganov@pppl.gov
}

cal approach is not valid and can overestimate the stripping cross sections by neutral atoms by an order-of-magnitude [6]. Therefore, a hybrid approach has been developed, which automatically chooses between the Born approximation and the classical mechanics approximation depending on the parameters of the collision [2].

\section{STRIPPING CROSS SECTIONS AT LARGE PROJECTILE VELOCITIES}

We have investigated theoretically and experimentally the stripping of 3.4 Mev/amu $K r^{+7}$ and $X e^{+11}$ in $N_{2}$ [4]; and 10.2 MeV/amu $A r^{+6}, 19 \mathrm{MeV} / \mathrm{amu} A r^{+8}, 30 \mathrm{MeV}$ $\mathrm{He}^{+}$, and $38 \mathrm{MeV} / \mathrm{amu} \mathrm{N}^{+6}$, all in $\mathrm{He}, \mathrm{N}_{2}, \mathrm{Ar}$ and $\mathrm{Xe}$ [5]. Data for $\mathrm{He}^{+}$, and $\mathrm{N}^{+6}$ are shown in Tables 1 and 2, respectively, together with the theoretical estimates. Here, the label BA refers to the theoretical calculations made using the Born approximation (described in detail in Appendix A of Ref.[1]), and CT refers to the theoretical calculations made using a quasi-classical model (described in detail in Appendix B of [1]); and the label Hybrid is a combination of both as described below. Note that the cross sections calculated in the Born approximation agree better with the experimental data for light target atoms, whereas the quasi-classical model results agree better with the experimental data for xenon. The disagreement between the calculations and the experimental data is due to the application of approximations outside their validity range.

To overcome this shortcoming we have developed the "hybrid method". The cross section can be represented as an integral over the impact parameter $\rho$. The simplest method to calculate the cross section is to use both the classical and Born approximations, but only for the regions in which they are valid, and sum the results to obtain the total cross section. But at what point is one method favored over the other? The range of validity of both approximations can be estimated by evaluating the action

$$
S(\rho)=\int_{-\infty}^{\infty} V[r(\rho, t)] d t
$$

along the trajectory $r(\rho, t)=\left[\rho^{2}+(v t)^{2}\right]^{1 / 2}$. At small impact parameters $\left(\rho<\rho^{*}\right)$, where $\rho^{*}$ is determined by the condition

$$
S\left(\rho^{*}\right)=\hbar,
$$

the target atom potential $V(r)$ is large and the corresponding change in action is large; thus, we can apply classical mechanics for $\rho<\rho^{*}$. Similarly, the Born approximation fails if $S(\rho)>\hbar$. Thus, as a natural cutoff, we shall use the 


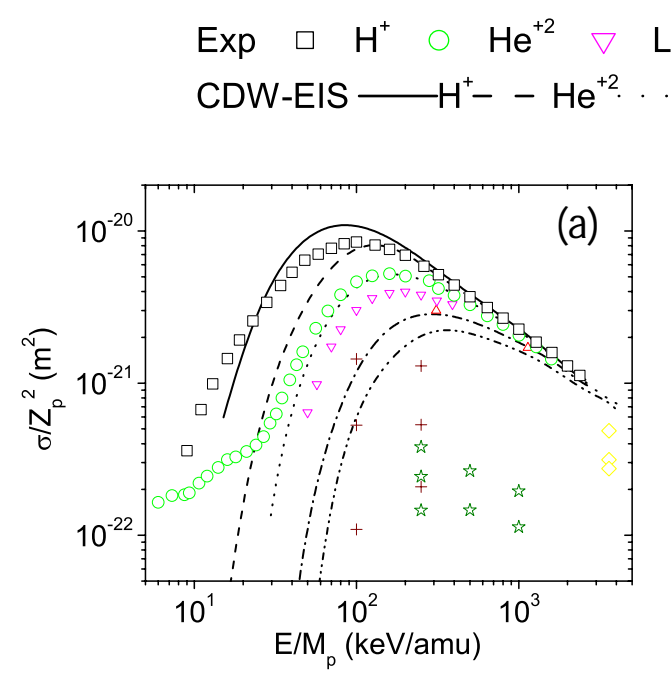

$$
\begin{aligned}
& \mathrm{Li}^{+3} \triangle \mathrm{C}^{+6} \mathrm{Au}^{+\mathrm{Z}_{\rho}}+\mathrm{I}^{+\mathrm{Z}_{\rho}} \text { 敢 } \mathrm{U}^{+\mathrm{Z}_{\rho}} \\
& \mathrm{Li}^{+3}--\mathrm{C}^{+6}-\cdots-\mathrm{O}^{+8} ; \text { Fit } \cdots \text { new }
\end{aligned}
$$

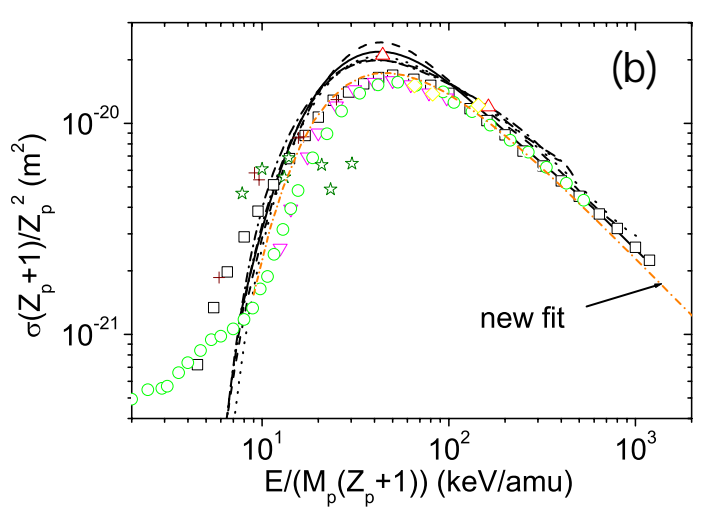

Figure 1: Ionization cross sections of helium by various stripped ions. The solid curves correspond to the theoretical calculation in the eikonal approximation, and the symbols label the experimental data (see text for details). Shown in the figures are: (a) the raw data, and (b) the scaled data making use of Eq.(3). The notation "new fit" refers to Eq.(4).

Born approximation for the regions where $S(\rho)<\hbar$, and we shall use the classical approach for the regions where $S(\rho)>\hbar$. The total cross section can then be expressed as a sum of the classical cross section in the region $\rho<\rho^{*}$, and the cross section calculated in the Born approximation in the region $\rho>\rho^{*}$.

Using the procedure outlined above, the cross sections presented in Table 1 have been recalculated. Results of the hybrid calculations are shown in the last columns of Tables 1 and 2. Note that the cross sections involving $\mathrm{He}$ as the target atom are identical for calculations making use of the Born approximation and for the calculations using the hybrid method. This is because $S(\rho) / \hbar$ is small for this parameter range, and the Born approximation remains valid throughout the entire region of integration. This suggests that the Born approximation should be more accurate than the classical approximation in this case. In the opposite case of xenon, the hybrid method gives results close to the calculations performed using classical mechanics, because $S(\rho) / \hbar$ remains larger than unity for most impact parameters, leading to ionization. In summary, the hybrid method helps to identify which method is more reliable. Moreover, it produces more reliable results than either of the approximations separately (see, for example, the case for Ar in Table 2).

The difference between the classical mechanics calculation and the Born approximation becomes more significant for fast projectiles [6]. As envisioned in heavy ion fusion, a driver will accelerate heavy ions up to energies of $25 \mathrm{MeV} / \mathrm{amu}$. For beam-plasma interaction issues it is important to evaluate stripping cross sections by neutral atoms and plasma ions. As a limiting case, we shall evaluate cross sections for projectiles with low ionization potentials colliding with neutral target atoms or fully stripped target ions. Table 3 shows the results of calculations of the stripping cross sections for only one electron from the outer electron shell for different projectile ions with the same velocity $v=32 v_{0}(25 \mathrm{MeV} / \mathrm{amu})$ colliding with a nitrogen atom $(N)$. At these energies $S(\rho) / \hbar$ remains much less than unity and the Born approximation is valid, whereas the classical mechanics calculation is not. For the experimental data in Tables 1 and 2, the difference between the two approximations is a factor 2 at most. We now study the difference for projectiles with much lower ionization potentials than in Tables 1 and 2. For example, $\mathrm{Cs}^{+}$has ionization potential $22.4 \mathrm{eV}, \mathrm{I}^{-}$has ionization potential $3 \mathrm{eV}$, and $H^{-}$has ionization potential $0.75 \mathrm{eV}$, whereas $\mathrm{He}^{+}$and $\mathrm{N}^{+6}$ have ionization potentials $54.4 \mathrm{eV}$ and $666 \mathrm{eV}$, respectively.

There is a large difference, up to a factor 10 (for $\mathrm{H}^{-}$), in the value of the stripping cross sections by neutral atoms calculated in the classical trajectory approximation and the Born approximation of quantum mechanics. The reason for this is the quantum mechanical uncertainty of the electron location. At high energies, classical mechanics overestimates the probability of ionization, presuming that the electron can be located at a prescribed impact parameter (see details in Ref. [6]).

\section{NEW FIT FORMULA FOR THE IONIZATION CROSS SECTION}

While specific values of the cross sections for various pairs of projectile ions and target atoms have been measured at several energies $[1,4,5,7]$, the study of the scaling of cross sections with energy and target or projectile nucleus is only now underway $[8,9]$. When experimental data and theoretical calculations are not available, approximate formulas are frequently used.

In Ref. [1], a new fit formula was proposed for the ion- 
Table 1: Stripping cross sections of $30 \mathrm{MeV} / \mathrm{amu} \mathrm{He}^{+}$in collisions with $\mathrm{He}, \mathrm{Ar}$ and $\mathrm{Xe}$. Exp. denotes the experimental data from [5]; BA denotes the cross sections calculated making use of the Born approximation of quantum mechanics; CT refers to calculations making use of classical mechanics only; and Hybrid is a combination of both models as described in the text.

\begin{tabular}{|l|c|c|c|c|}
\hline & Exp. & BA & CT & Hybrid \\
\hline$H e$ & 0.4 & 0.30 & 0.69 & 0.30 \\
\hline$A r$ & 7.3 & 9.0 & 11.5 & 9.1 \\
\hline$X e$ & 23. & 47 & 36 & 33 \\
\hline
\end{tabular}

Table 2: Similar to Table 1, but stripping cross sections are presented for $38 \mathrm{MeV} / \mathrm{amu} \mathrm{N}^{+6}$ in collisions with $\mathrm{He}, \mathrm{Ar}$ and $X e$.

\begin{tabular}{|c|c|c|c|c|}
\hline & Exp. & BA & CT & Hybrid \\
\hline$H e$ & 0.06 & 0.044 & 0.046 & 0.044 \\
\hline$A r$ & 1.64 & 1.58 & 1.58 & 1.68 \\
\hline$X e$ & 6.29 & 10.30 & 6.50 & 6.9 \\
\hline
\end{tabular}

ization cross section which has no fitting parameters. In particular, the following scaling was proposed

$$
\sigma^{i o n}\left(v, I_{n l}, Z_{p}\right)=\pi a_{0}^{2} \frac{N_{n l} Z_{p}^{2}}{\left(Z_{p}+1\right)} \frac{E_{0}^{2}}{I_{n l}^{2}} G\left(\frac{v}{v_{n l} \sqrt{Z_{p}+1}}\right),
$$

where $a_{0}$ is the Bohr radius, $Z_{p}$ is the projectile ion charge state, $E_{0}=27 \mathrm{eV}$ is the energy scale in atomic units, $I_{n l}$ is the ionization potential of an orbital with quantum numbers $n$ and $l$ and $v_{n l}=\sqrt{2 I_{n l} / E_{0}}$ is the characteristic atomic velocity, and $v$ is the projectile ion velocity. The fitting function in Eq.(3) is defined by

$$
G(x)=\frac{\exp \left(-x^{-2}\right)}{x^{2}}\left[1.26+0.283 \ln \left(2 x^{2}+25\right)\right]
$$

This formula has been checked against available experimental data and theoretical predictions. The most experimental data for ionization cross sections by various projectile ions was collected for helium and is shown in Fig.1.

Table 3: Stripping cross sections of $3.35 \mathrm{GeV} \mathrm{Cs}{ }^{+}$, $3.2 \mathrm{GeV} \mathrm{I}^{-}$and $25 \mathrm{MeV} \mathrm{H}^{-}$in collisions with $\mathrm{N}$ or $\mathrm{N}^{+7}$, calculated making use of the Born approximation (BA), and the classical trajectory approximation (CT). The stripping of only one electron from the outer electron shell is considered here with ionization potentials: $22.4 \mathrm{eV}$ for $\mathrm{Cs}^{+}$; $3.06 \mathrm{eV}$ for $\mathrm{I}^{-}$; and $0.75 \mathrm{eV}$ for $\mathrm{H}^{-}$. Hybrid is a combination of both models as described in the text.

\begin{tabular}{|c|c|c|c|}
\hline & BA & CT & Hybrid \\
\hline$C s^{+}$ & 0.045 & 0.10 & 0.045 \\
\hline$I^{-}$ & 0.08 & 0.47 & 0.08 \\
\hline$H^{-}$ & 0.10 & 1.34 & 0.10 \\
\hline
\end{tabular}

The symbols in Fig.1 denote the experimental data for $H^{+}, H e^{+2}, L i^{+3}$, for $C^{+6}$, for $I^{+Z_{p}}$ and $U^{+Z_{p}}$, and for $A u^{+Z_{p}}$, where $Z_{p}=10-40$ [1]. The solid curves in Fig.1 correspond to the continuum-distorted-wave-eikonal initial state (CDW-EIS) theoretical calculation. Further details are provided in Ref. [1].

\section{CONCLUSIONS}

The new scaling formulas in Eqs. (3) and (4) for the ionization and stripping cross sections of atoms and ions by fully stripped projectiles has been proposed. The scaling has no fitting parameters and has been checked against 11 different pairs of projectile and target atoms. For ionization cross sections of helium by 7 different ions presented in Fig.1, all of the experimental data overlap on a single curve in the proposed scaled coordinates.

We have developed a hybrid method for the calculation of the ionization and stripping cross sections of ions or atoms by fast ions by combining the Born approximation of quantum mechanics and the quasi-classical approach in the regions of impact parameters in which they are valid, and sum the results to obtain the total cross section. The hybrid method was checked against experimental data for more than 18 different pairs of projectile and target atoms in the range of 3-38 MeV/amu [2]. The results of the modified approach agree better with the experimental data than either the Born approximation or the quasi-classical approach, applied separately.

For fast projectile velocities and low ionization potentials, note that the classical approach is not valid and can overestimate the stripping cross sections by neutral atoms by an order-of-magnitude [6].

\section{REFERENCES}

[1] I. D. Kaganovich, E. A. Startsev and R. C. Davidson, "Formulary and scaling cross sections for ion-atom impact ionization", submitted to Phys. Rev. ST-AB (2004); http://arxiv.org/abs/physics/0407140; Physics of Plasmas 11, 1229 (2004).

[2] Igor D. Kaganovich, et. al., Nuclear Instruments and Methods in Physics Research A, in press (2005).

[3] R. K. Janev, L. P. Presnyakov and V. P. Shevelko, Physics of Highly Charged Ions (Springer, Berlin, 1999).

[4] D. Mueller, L. Grisham, I. Kaganovich, R. L. Watson, V. Horvat and K. E. Zaharakis, Physics of Plasmas 8, 1753 (2001).

[5] D. Mueller, et al., Laser and Particle Beams 20, 551 (2002).

[6] I. D. Kaganovich, E. A. Startsev and R. C. Davidson, Phys. Rev. A 68, 022707 (2003).

[7] R. E. Olson, R. L. Watson, V. Horvat and K. E. Zaharakis, J. Phys. B: At. Mol. Opt. Phys. 35, 1893 (2002).

[8] R. L. Watson, Y. Peng, V. Horvat, G. J. Kim and R. E. Olson, Phys. Rev. A 67, 022706 (2003).

[9] A.C.F. Santos and R.D. DuBois, Phys. Rev. A 69, 042709 (2004). 